\title{
ON THE ANOMALOUS BEHAVIOUR OF ANISOTROPIC SHEET METALS
}

\author{
BRADLEY DODD \\ Department of Engineering, University of Reading, Whiteknights, Reading, RG6 2AY, England \\ and \\ Robert M. CADDELL \\ Department of Mechanical Engineering and Applied Mechanics, The University of Michigan, \\ Ann Arbor, MI 48109, U.S.A.
}

(Received 2 April 1983)

\begin{abstract}
Summary-Using Hill's 1948 criterion[1] for anisotropic yielding and the strain ratio, $r$, it has been shown that the ratio of the balanced biaxial yield stress, $\sigma_{b}$, to the uniaxial tensile yield stress, $\sigma_{u}$, should be $>1$ if $r>1$ and $<1$ if $r<1$. Certain experimental results[2] showed that with commercial-purity aluminium, where $r<1$, the ratio of $\sigma_{b}$ to $\sigma_{u}$ was always $>1$ in that study. This was termed anomalous behaviour. Hill has proposed a new criterion [3] that not only appears to provide greater flexibility than does his earlier version but can also encompass anomalous behaviour which the earlier version cannot.

Four simplified cases of the 1979 criterion have been proposed [3] and to date only one has been subjected to experimental assessment. However, the goals of those studies were not concerned with anomalous behaviour per se. In this paper, all four cases are analysed to determine the interrelationships of the parameters $r$ and $m$ (exponent in Hill's new criterion) required to encompass anomalous behaviour. It is found that for each of the four cases anomalous behaviour is predicted for a range of $(m, r)$ combinations which are presented graphically in this paper.
\end{abstract}

\section{NOTATION}

$\sigma_{1}, \sigma_{2}, \sigma_{3}$ principal stresses

$F, G, H$ anisotropic parameters in Hill's 1948 yield criterion

$r$ strain ratio (width to thickness strains)

$r_{0}, r_{45}, r_{90}$ strain ratios at various angles to the rolling (zero) direction

$\bar{r}$ average strain ratio

$\sigma_{b}$ yield strength in balanced biaxial tension (in plane of sheet)

$\sigma_{u}$ yield strength in uniaxial tension (in plane of sheet)

$f, g, h, a, b, c$ anisotropic parameters in Hill's 1979 yield criterion

$\sigma$ scaling factor for stress

$m$ exponent in Hill's 1979 yield criterion

$A$ parameter equal to $\left(\sigma_{b} / \sigma_{u}\right)^{m}$

\section{INTRODUCTION}

In studies involving anisotropic sheet metals, it is necessary to employ a yield criterion. To date, Hill's 1948 criterion[1] has been more widely used than any other. In terms of principal stresses, this can be expressed as,

$$
F\left(\sigma_{2}-\sigma_{3}\right)^{2}+G\left(\sigma_{3}-\sigma_{1}\right)^{2}+H\left(\sigma_{1}-\sigma_{2}\right)^{2}=1
$$

where $F, G$, and $H$ are parameters that characterize the anisotropy and the $1,2,3$ directions correspond to the rolling, transverse, and through-thickness directions of a rolled sheet. As such, they correspond to the three principal axes of anisotropy.

In practice, equation (1) can be altered to utilize the ratios of width to thickness strains as measured under uniaxial tension in various directions in the plane of the sheet. Often, this $r$-value varies with orientation and an average value of this parameter has been applied in many cases. This average is defined by,

$$
\bar{r}=\frac{r_{90}+2 r_{45}+r_{0}}{4} \text {. }
$$


Once $\bar{r}$ is determined, it is then often used as if the sheet displayed planar isotropy. $\dagger$ If this is done, and a case of plane stress is considered $\left(\sigma_{3}=0\right)$, equation (1) can be simplified to,

$$
\sigma_{1}^{2}+\sigma_{2}^{2}-\frac{2 r}{(1+r)} \sigma_{1} \sigma_{2}=\sigma_{u}^{2}
$$

where $\sigma_{u}$ is the yield stress in uniaxial tension (often measured in the rolling direction). Since planar isotropy is assumed throughout this paper, we will use the symbol $r$ instead of $\bar{r}$ for simplicity.

Recently, Mellor [4] has given an excellent coverage of many aspects of forming with anisotropic sheet metals and it would appear that the use of equation (1), or any of its forms, does seem to be more acceptable with metals where $r>1$ than with some having $r<1$. Because our concern here is with so-called "anomalous behaviour", apparently first coined by Woodthorpe and Pearce[2] and the recent suggestions by Hill[3] to "encompass the anomaly", we make no attempt to document or discuss the many publications connected with other aspects of this broad question.

\section{ANOMALOUS BEHAVIOUR}

Woodthorpe and Pearce[2], using commercial-purity aluminum (annealed as well as in varying degrees of pre-strain), found that although $r$ ranged from about 0.48 to 0.65 , the ratio of yield strength in balanced biaxial tension, $\sigma_{b}$, to that in uniaxial tension, $\sigma_{u}$ was always $>1$. Both of these yield strengths were measured in the plane of the sheet. Now equation (1) or (3) can be revised to show that,

$$
\left(\sigma_{h} / \sigma_{u}\right)^{2}=\frac{1+r}{2}
$$

It is obvious that,

$$
\left(\sigma_{b} / \sigma_{u}\right)>1 \text { if } r>1
$$

and

$$
\left(\sigma_{b} / \sigma_{u}\right)<1 \text { if } \quad r<1
$$

but since, in the above study, $r<1$ and $\left(\sigma_{b} / \sigma_{u}\right)>1$, these authors [2] termed this "anomalous behaviour" and that term has often been used since that time. $\S$

\section{HILL'S 1979 CRITERION [3]}

In an attempt to provide a criterion of possibly broader applicability than equation (1), and, to "encompass the anomaly", Hill has proposed the following anisotropic yield criterion,

$$
\begin{gathered}
f\left|\sigma_{2}-\sigma_{3}\right|^{m}+g\left|\sigma_{3}-\sigma_{1}\right|^{m}+h\left|\sigma_{1}-\sigma_{2}\right|^{m}+a\left|2 \sigma_{1}-\sigma_{2}-\sigma_{3}\right|^{m} \\
+b\left|2 \sigma_{2}-\sigma_{3}-\sigma_{1}\right|^{m}+c\left|2 \sigma_{3}-\sigma_{1}-\sigma_{2}\right|^{m}=\sigma^{m}
\end{gathered}
$$

where the six coefficients $(f, g$, etc.) characterize the anisotropy, $\sigma$ is a scaling factor for the stress, and $m>1$ to insure convexity. If $m=2$ and the coefficients are rearranged, this reduces to equation (1). When $m \neq 2$ there are then seven parameters that can take on various values thereby affording greater flexibility compared to equation (1). This new criterion can also account for anomalous behaviour when certain parametric values are assigned.

Four special cases of equation (7) have been proposed by Hill, all being based upon plane stress and planar isotropy. As he has stated, these are not the only possible cases but until these are tested against experiment, it would be premature to propose others. To assist the reader, we will include all assumptions involved. They are,

$$
\begin{array}{ll}
\text { Case (i) } & a=b=h=0, f=g \\
\text { Case (ii) } & a=b, c=f=g=0 \\
\text { Case (iii) } & a=b, f=g, c=h=0 \\
\text { Case (iv) } & a=b=f=g=0 .
\end{array}
$$

$\dagger$ Technically this is not correct even though this has, on occasion, provided good correlation between prediction and experimental results.

†Hosford and Backofen [5] introduced the terms "texture hardening" if $r>1$ and "texture softening" if $r<1$.

$\$$ Hosford et al.[6-8] suggested a modification of equation (1) based upon crystallographic calculations. However, like equation (1) it cannot account for anomalous behaviour. 
Using these sequentially in equation (7) results in,

$$
\begin{array}{ll}
\text { Case (i) } & c\left|\sigma_{1}+\sigma_{2}\right|^{m}+f\left(\left|\sigma_{1}\right|^{m}+\left|\sigma_{2}\right|^{m}\right)=\sigma^{m} \\
\text { Case (ii) } & a\left(\left|2 \sigma_{1}-\sigma_{2}\right|^{m}+\left|2 \sigma_{2}-\sigma_{1}\right|^{m}\right)+h\left|\sigma_{1}-\sigma_{2}\right|^{m}=\sigma^{m} \\
\text { Case (iii) } & a\left(\left|2 \sigma_{1}-\sigma_{2}\right|^{m}+\left|2 \sigma_{2}-\sigma_{1}\right|^{m}\right)+f\left(\left|\sigma_{1}\right|^{m}+\left|\sigma_{2}\right|^{m}\right)=\sigma^{m} \\
\text { Case (iv) } & c\left|\sigma_{1}+\sigma_{2}\right|^{m}+h\left|\sigma_{1}-\sigma_{2}\right|^{m}=\sigma^{m}
\end{array}
$$

The following were also developed [3],

$$
r=\frac{a\left(2^{m-1}+2\right)-c+h}{a\left(2^{m-1}-1\right)+2 c+f}
$$

and

$$
\left(\sigma_{b} / \sigma_{u}\right)^{m}=\frac{1+r}{2}\left\{1+\frac{\left(2^{m-1}-2\right)(a-c)}{a+c 2^{m-1}+f}\right\} .
$$

If the parameters proposed in equation (8) are introduced in (10) and (11), the four cases result in,

$$
\begin{array}{ll}
\text { Case (i) } & \left(\sigma_{b} / \sigma_{u}\right)^{m}=(1+r) /\left\{2+r\left(4-2^{m}\right)\right\} \\
\text { Case (ii) } & \left(\sigma_{b} / \sigma_{u}\right)^{m}=\left\{2^{m-1}(1+r)-(1+r)\right\} / 2 \\
\text { Case (iii) } & \left(\sigma_{b} / \sigma_{u}\right)^{m}=\left\{(1+r)\left(2+2^{m-1}\right)\right\} /\left\{4(1+r)+2^{m}(1-r)\right\} \\
\text { Case (iv) } & \left(\sigma_{b} / \sigma_{u}\right)^{m}=(1+r) /\left(2^{m-1}\right) .
\end{array}
$$

To investigate anomalous behaviour (hence we are concerned only with the situation that $\left(\sigma_{b} / \sigma_{u}\right)>1$ when $r<1$ ), requires the following,

$$
\begin{array}{ll}
\text { Case (i) } & (1+r)>\left(2+4 r-r 2^{m}\right) \text { or }\left\{r\left(3-2^{m}\right)+1\right\}<0 \\
\text { Case (ii) } & \left\{2^{m-1}(1+r)-(1+r)\right\}>2 \text { or }(3+r)-2^{m-1}(1+r)<0 \\
\text { Case (iii) } & \left\{(1+r)\left(2-2^{m-1}\right)+2^{m}(1-r)\right\}<0 \\
\text { Case (iv) } & 2^{m-1}-(1+r)<0 .
\end{array}
$$

For all cases, particular values of $m$ and $r$ would encompass anomalous behaviour; these cases are now discussed individually.

\section{CASE I}

Here we start with equation (12a) and note that as $\left(\sigma_{b} / \sigma_{u}\right) \rightarrow 1$ (lowest bound on this ratio) then, in the limit,

$$
(1+r)=2+4 r-r 2^{m} \text { or } 2^{m}=(3 r+1) / r .
$$

Considering the values of $m$ for $0.1 \leqslant r \leqslant 0.9$, the effect of $r$ on $m$ is shown in Fig. 1 noting that the range of $r$ chosen is arbitrary but would appear to include reported results for metals when $r<1$. Maximum values of $m$, also shown in that figure, were found by letting $\left(\sigma_{b} / \sigma_{u}\right) \rightarrow \infty$ (obviously this would never occur in reality so the computed $m$-values must be viewed as being larger than would ever be expected in practice). This requires,

$$
2+4 r-r 2^{m}=0 \text { or } 2^{m}=(4 r+2) / r .
$$

Thus, for this case, as $r$ varies as indicated, $m$ can be definitely bounded. Note that as $r \rightarrow 1, m \rightarrow 2$ in the limit of the stress ratio, $\left(\sigma_{b} / \sigma_{u}\right)$, approaching unity.

\section{CASE II}

We note here that Cases ii-iv, one bounding of $m$ using $\left(\sigma_{b} / \sigma_{u}\right) \rightarrow \infty$ will not work as with Case $\mathrm{i}$ and the reasons will be pointed out for each. Using equation (12b) and letting the stress ratio approach unity leads to,

$$
2^{m}=2(3+r) /(1+r) .
$$

The variation of $m$ with $r$ is shown in Fig. 2, again noting that as $r \rightarrow 1, m \rightarrow 2$. To find an upper bound, and letting $A=\left(\sigma_{b} / \sigma_{u}\right)^{m}$, we have,

$$
2^{m}=4 A /(1+r)+2
$$

Two approaches present themselves, either a fixed value can be assigned to $A$ or to $\left(\sigma_{b} / \sigma_{u}\right)$. We have chosen the latter using an arbitrary value of 1.2 for the stress ratio and we note that in this case, if $A$ were taken as 2 , only slightly higher values result for $m$ as a function of $r$ (of course if $A$ is fixed and $m$ then found, the stress ratio varies with $r$. Here if $A=2$, that ratio varies from 1.24 with $r=0.1$ to 1.31 for $r=0.9$ ). The results, using $\sigma_{b} / \sigma_{u}=1.2$ are shown on Fig. 2.

$\dagger$ These result after rearrangement of terms. 


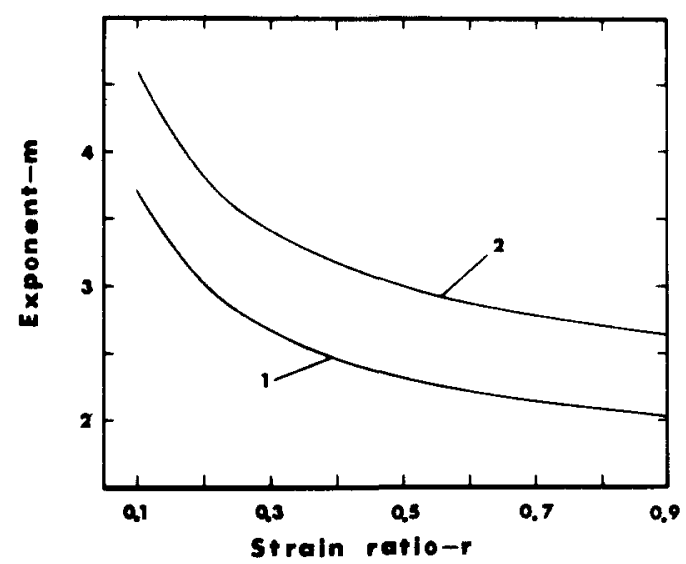

FiG. 1. Values of $m$ as a function of $r$ to encompass anomalous behaviour based upon Case i of equation (7). Curve 1 results when $\left(\sigma_{b} / \sigma_{u}\right)=1$ while curve 2 results when $\left(\sigma_{b} / \sigma_{u}\right) \rightarrow \infty$.

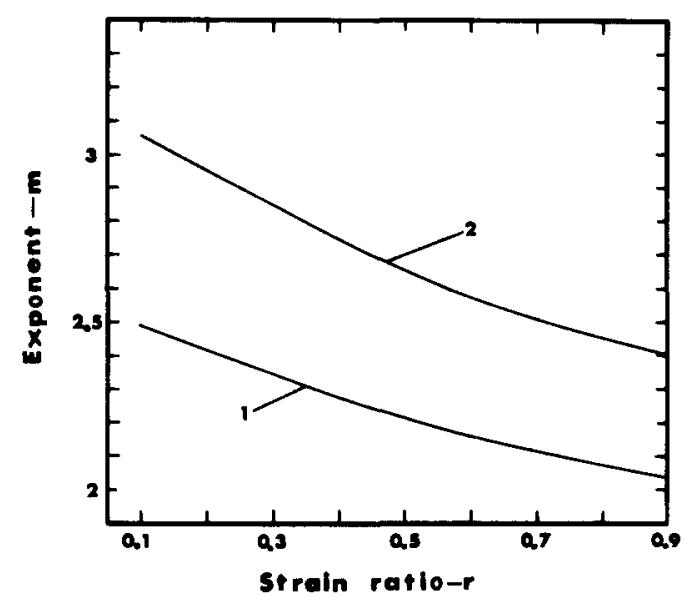

FIG. 2. Values of $m$ as a function of $r$ to encompass anomalous behaviour based upon Case ii of equation (7). Curve 1 is for $\left(\sigma_{b} / \sigma_{u}\right)=1$ while curve 2 is for $\left(\sigma_{b} / \sigma_{u}\right)=1.2$.

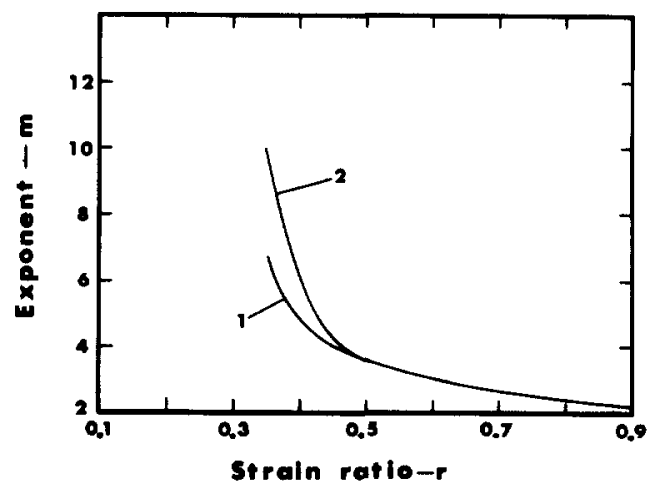

FIG. 3. Same as Figs. 1 and 2 using Case iii of equation (7). Curve 1 for $\left(\sigma_{b} / \sigma_{u}\right)=1$ while curve 2 is for $\left(\sigma_{b} / \sigma_{u}\right)=1.0035$ ! 


\section{CASE III}

With equation $(12 \mathrm{c})$ and with $\left(\sigma_{b} / \sigma_{u}\right) \rightarrow 1$, there results,

$$
2^{m}=4(1+r) /(3 r-1) .
$$

Since real values of $m$ result only in $r>1 / 3$, a lower limit of $r=0.35$ was used for illustrative purposes. Fig. 3 shows how $m$ varies with $r$ from equation (18). For stress ratios $>1$ and letting $A=\left(\sigma_{b} / \sigma_{u}\right)^{m}$, equation (12c) can be expressed as,

$$
A=\frac{2(1+r)+2^{m}(1+r) / 2}{4(1+r)+2^{m}(1-r)} .
$$

Here, for $A>1$ and $m$ to be real, $(1+r) / 2$ in the numerator must be $>(1-r)$ in the denominator, so for a given value of $r$, the ratio of $(1+r) / 2(1-r)$ defines a maximum value for $A$. This ranges from 1.038 to 9.5 as $r$ varies from 0.35 to 0.9 . What this infers is that if a constant value of $\left(\sigma_{b} / \sigma_{u}\right)$ is to be assumed, it must be based upon the case where $r=0.35$. Calculations indicated that a stress ratio just barely greater than unity must be used (we have chosen 1.0035). The results are shown in Fig. 3 and except at lower values of $r$, the variation of $m$ with $r$ is practically identical with those based upon equation (18) as would be expected. It is important to realize that if a larger stress ratio, say 1.2, were used instead of 1.0035 then real values of $m$ would not be predicted if $r$ were $<0.4$ or thereabouts. Fig. 3 shows the effect of $r$ on $m$ when $\left(\sigma_{b} / \sigma_{u}\right)=1.0035$ and indicates the extreme sensitivity of this stress ratio on $m$.

Using the maximum value of $A$ as a function of $r$, (equation 19), is not very satisfactory since the plot of $m$ versus $r$ oscillates. In addition, as an extreme example, if for $r=0.9$ and an acceptable limit of $A=9.4$ were used (equation 19), the predicted value for $m$ is 12.72, yet the corresponding stress ratio is only 1.19. What this seems to imply is that the form of this case not only limits a minimum possible value for $r$ but also that very low stress ratios must be used (barely $>1$ ), if the full range of the effect of $r$ on $m$ is to be portrayed.

\section{CASE IV}

With equation (12d) and letting the stress ratio approach unity, there results,

$$
2^{m}=2(1+r)
$$

and Fig. 4 shows the results; again as $r \rightarrow 2$.

Considering the other bound and using $A$ in its usual context, equation (12d) can be expressed as,

$$
A=2(1+r) / 2^{m} .
$$

Now since $m>1$, the maximum value of $A$ with $r$ is $(1+r)$. Calculations show that if this value were used as $r$ varied, the minimum stress ratio that would lead to required values of $m>1$ for any $r$, is about 1.1 . This was used in equation (21) and the results are also shown on Fig. 4. It is noted that this is the only case where the use of unity for the stress ratio provides an upper bound on $m$. Note that because of the ranges of $m$ involved, the scale of the ordinate differs on the four figures.

\section{COMPARISON WITH EXPERIMENTAL DATA}

Hill[3] has quoted the data listed in Table 1 from the references indicated. It is our understanding that only Case iv (i.e. equation 12d) were used to determine the values of $m$ indicated in that table. If $\left(\sigma_{b} / \sigma_{u}\right)>1$ in all of those situations, the following may be noted:

(1) Fig. 4 shows excellent agreement with the data in Table 1, as of course it must since equation (12d) was used.

(2) Using this same equation indicates that data other than that attributed to Woodthorpe and Pearce also displays the "anomaly".

(3) If Cases i to iii were chosen as yield and plastic potential functions, then values of $m>2$ are required to predict anomalous behaviour.

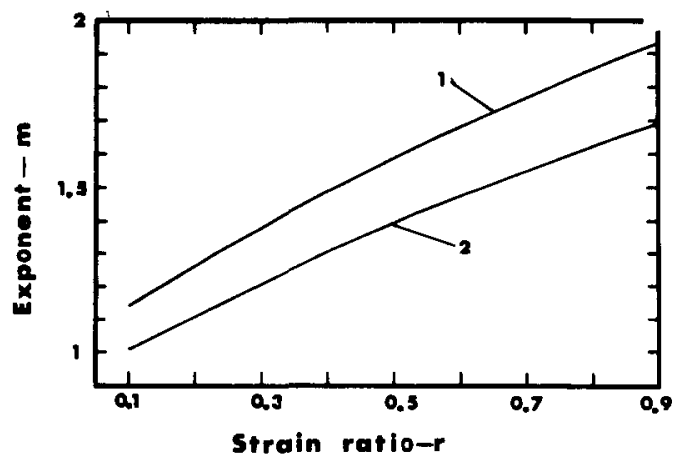

Fig. 4. Same as Figs. 1 and 2 using Case iv of equation (7). Curve 1 for $\left(\sigma_{b} / \sigma_{u}\right)=1$ while curve 2 is for $\left(\sigma_{b} / \sigma_{u}\right)=1.1$. 
TABle 1. Experimental Values of $r$ AND $m$

\begin{tabular}{llcll}
\hline $\mathrm{r}$ & $\mathrm{m}$ & Reference & *Predicated Behaviour & Material \\
\hline 0.6 & 1.47 & 2 & Anomalous & Aluminium \\
0.5 & 1.38 & 2 & Anomalous & Aluminium \\
0.44 & 1.5 & 9 & Anomalous & Rirming Steel \\
0.72 & 1.8 & 9 & Borderline & Soft Alumini um \\
0.63 & 1.7 & 10 & Borderline & Soft Aluminium \\
0.86 & 1.8 & 10 & Anomalous & $70 / 30$ Brass \\
\hline
\end{tabular}

*Using either Equation (12d) or (13d).

\section{DISCUSSION}

To date, only one version of equation (7) appears to have been compared with experimental results [9-11]; this is equation (9d) or Case iv. Those studies were not, in themselves, primarily concerned with possible anomalous behaviour. Since there is simply not enough evidence that has yet accrued to fully assess the merits of equation (9d), it seems likely that the other three proposed cases (i.e. equations 9a-9c) will eventually be compared with experimental results.

If, in such studies, anomalous behaviour is observed, the approaches that led to Figs. 1-4 should prove helpful, at least that is our major intent in presenting this paper. We especially point out that Case iii (as expressed by equation 19) seems to require special attention since its use is limited to a minimum value of $r$. Additionally, rather large values of $m$ would be required. Perhaps in a fortuitous sense, they are similar in magnitude to those proposed by Hosford et al. [6,7] albeit from different reasoning. The major difference is that while equation (12c) can account for anomalous behaviour, Hosford's form cannot.

\section{CONCLUSIONS}

All four special cases of Hill's 1979 anisotropic yield criterion can encompass anomalous behaviour for sheet metals having $r<1$ if proper values are assigned to the exponent $m$. Although only one of the four cases has been tested to date, that case indicates that such behaviour may not be restricted to the data of Woodthorpe and Pearce. As Hill has mentioned, such behaviour may not be uncommon.

Acknowledgements - The authors thank Prof. A. G. Atkins for his interest and helpful comments. Bradley Dodd is the Metal Box Research Fellow in the University of Reading and wishes to acknowledge the financial support of Metal Box p.l.c.

\section{REFERENCES}

1. R. Hill, Proc. Roy. Soc. London A 193, 281 (1948).

2. J. WoOdThORPE and R. PearCe, Int. J. Mech. Sci. 12, 341 (1970).

3. R. Hill, Math. Proc. Cambr. Phil. Soc. 85, 179 (1979).

4. P. B. MeLlOR, Mechanics of Solids, The Rodney Hill 60th Anniversary, (Edited by H. G. Hopkins and M. J. Sewell), p. 383. Pergamon Press, Oxford (1982).

5. W. F. HOSFORD and W. A. BACKOFEN, Fundamentals of deformation processing. Sagamore Army Matls. Res. Conf., Syracuse, New York, p. 259. Syracuse University Press (1964).

6. W. F. Hosford, 7th North Amer. Res. Conf. Proc., p. 191. SME, Dearborn, Michigan (1979).

7. R. Logan and W. F. Hosford, Int. J. Mech. Sci. 22, 419 (1980).

8. W. F. Hosford and R. M. CADDell, Metal Forming: Mechanics and Metallurgy, p. 269. Prentice-Hall, Englewood Cliffs, New Jersey (1983).

9. A. Parmer and P. B. Mellor, Int. J. Mech. Sci. 20, 385 (1978).

10. A. Parmer and P. B. Mellor, Int. J. Mech. Sci. 20, 707 (1978).

11. C. Vial, W. F. Hosford and R. M. CAdDell, Int. J. Mech. Sci., in press. 